\title{
Efeito da luz e da temperatura na germinação de sementes de quatro espécies de Xyris L. (Xyridaceae) ocorrentes na Serra do Cipó, MG, Brasil ${ }^{1}$
}

\author{
Maria Elizabeth Pereira Abreu e Queila Souza Garcia2,3
}

Recebido em 13/04/2004. Aceito em 06/08/2004

\begin{abstract}
RESUMO-(Efeito da luz e da temperatura na germinação de sementes de quatro espécies de Xyris L. (Xyridaceae) ocorrentes na Serra do Cipó, MG, Brasil). As espécies do gênero Xyris são típicas de solos brejosos ou úmidos, sendo bastante freqüentes nos campos rupestres de Minas Gerais. O objetivo deste estudo foi investigar o comportamento germinativo de sementes de $X$. cipoensis Smith \& Downs, $X$. longiscapa A. Nilsson, X. platystachia A. Nilsson e X. trachyphylla Mart. sob diferentes condições de luz e temperatura. Os experimentos de germinação foram realizados em câmaras de germinação nas temperaturas constantes de $15,20,25,30,35$ e $40^{\circ} \mathrm{C}$, sob luz e escuro contínuos, e nas alternâncias de 25-15, 30-15, 30-20, 35-15, 35-20 e 35-25ㅇ $\mathrm{C}$, onde as temperaturas mais altas referem-se ao período de luz, sob fotoperíodo de 12 horas. As quatro espécies de Xyris apresentam sementes pequenas e sensíveis à luz, com resposta nula de germinação no escuro. As sementes de X. cipoensis germinaram em faixa mais estreita de temperatura $\left(20\right.$ a $\left.30^{\circ} \mathrm{C}\right)$, apresentando alta porcentagem de germinação na temperatura constante de $20^{\circ} \mathrm{C}$. A faixa de 15 a $30^{\circ} \mathrm{C}$ foi favorável à germinação das sementes de $X$. longiscapa, X. platystachia e X. trachyphylla, apresentando baixo percentual de germinação a $15^{\circ} \mathrm{C}$. As temperaturas alternantes não favoreceram a germinação em relação às temperaturas constantes.
\end{abstract}

Palavras-chave: germinação, tamanho de semente, fotoblastimo positivo, temperatura, Xyris

ABSTRACT - (Light and temperature effect on germination of four species of Xyris L. (Xyridaceae) seeds occurring at the Serra do
Cipó, MG, Brazil). The Xyris genus species are typical from marshy or wet soils, being quite common on Minas Gerais rocky fields. The
objective of this study was to investigate the germinative behavior of X. cipoensis Smith \& Downs, X. longiscapa A. Nilsson, X.
platystachia A. Nilsson and $X$. trachyphylla Mart. under different light and temperatures conditions. The germination experiments took
place in germination chambers at constant temperatures of $15,20,25,30,35$ and $40^{\circ} \mathrm{C}$, under continuous white light and darkness, and
on the alternating temperatures of $25-15,30-15,30-20,35-15,35-20$ and $35-25^{\circ} \mathrm{C}$, the higher temperature being in light at a 12 hours
photoperiod. The four species of $X y$ ris have small and light sensitive seeds, with no germination on darkness. The seeds of $X$. cipoensis
germinated in a strict temperature $\left(20\right.$ to $\left.30^{\circ} \mathrm{C}\right)$, presenting higher percentage of germination at the constant temperature of $20^{\circ} \mathrm{C}$. The 15
to $30^{\circ} \mathrm{C}$ range were favorable to the germination of $X$. longiscapa, X. platystachia and $X$. trachyphylla seeds. These species presented low
percentage of germination at $15^{\circ} \mathrm{C}$. The alternating temperature did not favor the germination when compared to the constant temperatures.

Key words: germination, seed size, positive photoblastic, temperature, Xyris

\section{Introdução}

O conhecimento da biologia das sementes é essencial para a compreensão dos processos de estabelecimento, sucessão e regeneração natural da vegetação (Vázquez-Yanes \& Orozco-Segovia 1993). As sementes respondem a combinações específicas de luz, temperatura, umidade e concentrações de gases que são mais favoráveis para o estabelecimento da plântula (Baskin \& Baskin 1988; Ghersa et al. 1992). Luz e temperatura são importantes fatores ambientais controladores de germinação e dormência, tendo um papel crucial na regulação do crescimento e desenvolvimento das plantas (Baskin \& Baskin 1988; Pons 1992; Probert 1992). Segundo Labouriau (1983), a distribuição geográfica de muitas plantas é determinada, entre outros fatores, pela faixa de condições ambientais toleradas para a germinação de suas sementes. Dessa forma, estudos que abordam a fisiologia da germinação podem contribuir para explicar peculiaridades biogeográficas de espécies nativas, permitindo o entendimento do estabelecimento destas plantas em determinado habitat.

A família Xyridaceae é formada por plantas herbáceas, em sua maioria perenes e acaules, que ocorrem preferencialmente em solos brejosos e úmidos

\footnotetext{
Parte da Dissertação de Mestrado da primeira Autora

2 Universidade Federal de Minas Gerais, Departamento de Botânica, Fisiologia Vegetal, ICB, C. Postal 486, CEP 30161-970, Belo Horizonte, MG, Brasil

3 Autor para correspondência: queila@dedalus.lcc.ufmg.br
} 
das regiões tropicais e subtropicais (Smith \& Downs 1968). O gênero Xyris L. encontra-se amplamente distribuído nos campos rupestres do Brasil, sendo bastante freqüente na Cadeia do Espinhaço e Serra Geral de Goiás (Giulietti et al. 1988). Nos campos rupestres da Serra do Cipó ocorrem 42 espécies de Xyris, além de 4 variedades e 1 subespécie, sendo 14 endêmicas (M.G.L. Wanderley, comunic. pessoal). Em Minas Gerais, 16 espécies de Xyris estão listadas como ameaçadas e presumivelmente ameaçadas de extinção (Mendonça \& Lins 2000). Entre estas estão Xyris cipoensis e X.platystachia, devido à coleta indiscriminada das inflorescências, que são comercializadas como sempre vivas, e por ocorrerem em pequenas populações de distribuição restrita (Giulietti et al. 1996; Mendonça \& Lins 2000). A coleta é sempre feita antes do desenvolvimento dos frutos, o que afeta sensivelmente a reprodução sexuada e compromete o recrutamento natural das espécies (Giulietti et al. 1988; Kraus et al. 1994; Giulietti et al. 1996).

Estudos com Xyridaceae têm enfocado aspectos morfológicos e anatômicos (Kraus et al. 1994; Sajo et al. 1995; Rudall \& Sajo 1999), florísticos e taxonômicos (Smith \& Downs 1968; Giulietti et al. 1996). Tendo em vista a representatividade da família neste ecossistema e a escassez de informações sobre o ciclo de vida dessas espécies, o objetivo deste estudo foi verificar a influência de temperaturas constantes e alternadas, combinadas à presença ou ausência de luz, na germinação de espécies de Xyris simpáticas da Serra do Cipó, visando fornecer informações sobre os padrões de germinação, contribuindo para o desenvolvimento de um plano de manejo para a conservação destas espécies.

\section{Material e métodos}

A Serra do Cipó está situada na porção Sul da Cadeia do Espinhaço, no Estado de Minas Gerais, Brasil. O clima dessa região é do tipo tropical de altitude, com verões chuvosos e invernos secos, com média pluvial anual de $1.600 \mathrm{~mm}$ e temperatura média anual de $21^{\circ} \mathrm{C}$ (Marques et al. 2000). Frutos maduros $(\mathrm{n}=50$ para cada espécie) de Xyris cipoensis Smith \& Downs e Xyris platystachia Alb. Nilsson foram coletados em populações localizadas em áreas adjacentes ao Parque Nacional da Serra do Cipó (19 $15^{\circ}$ 'S, 433' 'W) e de Xyris longiscapa Alb. Nilsson e Xyris trachyphilla Mart. no Parque Nacional da Serra do Cipó (19² $14^{\prime}$ S, $\left.43^{\circ} 20^{\prime} \mathrm{W}\right)$. No laboratório, as sementes foram retiradas dos frutos e imediatamente utilizadas nos experimentos de germinação.

As medidas de comprimento e largura das sementes foram obtidas com auxílio de um paquímetro digital, utilizando uma amostragem de 100 sementes por espécie. Devido ao reduzido tamanho das sementes, a massa seca e o teor de água das sementes foram determinados a partir da pesagem inicial de 4 repetições de 100 sementes por espécie. As sementes foram colocadas em estufa a $105^{\circ} \mathrm{C}$ até a estabilização do peso, resfriadas à temperatura ambiente em dessecador e repesadas para obtenção da massa seca. Posteriormente, foi estimada a massa por semente e o teor de água foi expresso com base na massa fresca.

Os testes de germinação foram realizados utilizando-se placas de Petri de $9 \mathrm{~cm}$ diâm., contendo folha dupla de papel filtro umedecidas com água destilada, em 4 repetições de 25 , totalizando 100 sementes por tratamento. Os testes foram realizados em câmaras com luz e temperatura controladas. As placas foram colocadas sob luz branca $\left(30 \mu \mathrm{mol} \mathrm{m}^{-2} \mathrm{~s}^{-1}\right)$ e escuro contínuos, sob as temperaturas constantes de $15,20,25,30,35$ e $40^{\circ} \mathrm{C}$. No tratamento escuro as placas de Petri foram envolvidas com papel alumínio e mantidas em sacos pretos de polietileno. Também foi avaliada a germinação em temperaturas alternadas de $25-15,30-15,30-20,35-15,35-20$ e $35-25^{\circ} \mathrm{C}$, sob fotoperíodo de doze horas, onde as temperaturas altas se referem ao período de luz. A contagem das sementes germinadas foi realizada diariamente, utilizando-se microscópio estereoscópico e luz verde de segurança. O critério de germinação foi à emergência da radícula.

Os dados obtidos foram analisados pelo teste Brown-Forsythe (Zar 1996) para verificação da homogeneidade. Como os dados de germinabilidade apresentaram desvios padrões com variação errática, não sujeitos a normalização, realizou-se a análise não paramétrica de Kruskal-Wallis, cujas ordenações médias foram então comparadas com o teste $t$ de Student, ao nível 5\% de significância (Sampaio 2002). O tempo médio de germinação foi calculado segundo Labouriau (1983) e submetidos à análise de variância simples e comparados pelo teste de Tukey ao nível $5 \%$ de significância.

\section{Resultados}

As quatro espécies de Xyris apresentam sementes pequenas e leves, sendo maiores as medidas e os teores de água obtidos para as sementes de $X$. cipoensis e $X$. platystachia (Tab. 1). 
Tabela 1. Medidas de comprimento, largura, massa seca e teor de água de sementes de Xyris L. (média \pm erro padrão).

\begin{tabular}{|c|c|c|c|c|}
\hline Espécie & $\begin{array}{l}\text { Comprimento }(\mathrm{mm}) \\
\mathrm{n}=100\end{array}$ & $\begin{array}{l}\text { Largura }(\mathrm{mm}) \\
\quad \mathrm{n}=100\end{array}$ & $\begin{array}{c}\text { Massa Seca }(\mathrm{mg}) \\
\mathrm{n}=400\end{array}$ & $\begin{array}{c}\text { Teor de água (\%) } \\
\mathrm{n}=400\end{array}$ \\
\hline X. cipoensis & $1,06 \pm 0,22$ & $0,37 \pm 0,04$ & $0,0100 \pm 0,0002$ & $14,3 \pm 0,6$ \\
\hline X. longiscapa & $0,56 \pm 0,08$ & $0,19 \pm 0,02$ & $0,0019 \pm 0,0002$ & $12,7 \pm 4,1$ \\
\hline X. platystachia & $0,92 \pm 0,10$ & $0,33 \pm 0,05$ & $0,0075 \pm 0,0008$ & $14,2 \pm 3,2$ \\
\hline X. trachyphylla & $0,66 \pm 0,08$ & $0,22 \pm 0,03$ & $0,0031 \pm 0,0002$ & $11,5 \pm 2,0$ \\
\hline
\end{tabular}

As sementes das quatro espécies de Xyris necessitam de luz para iniciarem o processo de germinação, com resposta nula de germinação quando mantidas no escuro, o que as caracteriza como fotoblásticas positivas.

As sementes de $X$. cipoensis apresentaram maior porcentagem de germinação a $20^{\circ} \mathrm{C}$, sendo a germinação significativamente reduzida nas temperaturas constantes de 25 e $30^{\circ} \mathrm{C}$ (Tab. $2 \mathrm{~A}$ ). A germinabilidade foi semelhante entre as alternâncias $30-20$ e $30-15^{\circ} \mathrm{C}$ e inferior a $25-15^{\circ} \mathrm{C}$ (Tab. 2B). As alternâncias $30-15$ e $30-20^{\circ} \mathrm{C}$ promoveram a germinação como as temperaturas constantes de $20 \mathrm{e}$ $25^{\circ} \mathrm{C}(\mathrm{P}<0,05)$.

A germinabilidade das sementes de $X$. longiscapa foi semelhante nas temperaturas de 20 e $25^{\circ} \mathrm{C}$, com menor percentual de germinação a $15^{\circ} \mathrm{C}$ (Tab. $\left.2 \mathrm{~A}\right)$. As sementes de $X$. longiscapa apresentaram maior porcentagem de germinação na temperatura alternada de $30-20^{\circ} \mathrm{C}$, sendo a germinação significativamente reduzida nas alternâncias de $30-15$ e $25-15^{\circ} \mathrm{C}$ (Tab. 2B). Sob os regimes alternados de $30-15$ e $25-15^{\circ} \mathrm{C}$ as sementes apresentaram percentuais de germinação similares à temperatura constante de $15^{\circ} \mathrm{C}(\mathrm{P}<0,05)$.

As sementes de $X$. platystachia apresentaram porcentagem de germinação sem diferença significativa nas temperaturas de 20 e $25^{\circ} \mathrm{C}$ e percentual inferior a 15 e $30^{\circ} \mathrm{C}$ (Tab. $\left.2 \mathrm{~A}\right)$. Os valores de porcentagem de germinação não apresentaram diferenças significativas entre si nas alternâncias $25-15,30-15$ e $30-20^{\circ} \mathrm{C}$ (Tab. 2B) e entre as temperaturas constates 20 e $25^{\circ} \mathrm{C}$ $(\mathrm{P}<0,05)$.

A germinabilidade das sementes de $X$. trachyphylla não diferiu na faixa de temperatura de 20 a $30^{\circ} \mathrm{C}$, com valores inferiores a $15^{\circ} \mathrm{C}$ (Tab. $2 \mathrm{~A}$ ). As sementes de $X$. trachyphylla apresentaram maior

Tabela 2. Germinação média (\%) de sementes de Xyris L., submetidas a temperaturas constantes (luz branca contínua) (A) e alternantes (fotoperíodo de 12h/luz) (B). Letras iguais nas colunas não diferem entre si $(\mathrm{P} \leq 0,05)$.

A

\begin{tabular}{|c|c|c|c|c|}
\hline \multirow[b]{2}{*}{ Temperatura $\left({ }^{\circ} \mathrm{C}\right)$} & \multicolumn{4}{|c|}{ Espécie } \\
\hline & X. cipoensis & X. longiscapa & X.platystachia & X. trachyphylla \\
\hline \multicolumn{5}{|l|}{ Constante } \\
\hline 15 & $0 \mathrm{~d}$ & $54 \mathrm{c}$ & $76 b$ & $76 \mathrm{~b}$ \\
\hline 20 & $81 \mathrm{a}$ & $87 \mathrm{a}$ & $98 \mathrm{a}$ & $96 \mathrm{a}$ \\
\hline 25 & $66 \mathrm{~b}$ & $79 \mathrm{ab}$ & $96 \mathrm{a}$ & $92 \mathrm{a}$ \\
\hline 30 & $58 \mathrm{c}$ & $76 \mathrm{~b}$ & $86 \mathrm{ab}$ & $92 \mathrm{a}$ \\
\hline 35 & $0 \mathrm{~d}$ & $0 \mathrm{~d}$ & $0 \mathrm{c}$ & $0 \mathrm{c}$ \\
\hline 40 & $0 \mathrm{~d}$ & $0 \mathrm{~d}$ & $0 \mathrm{c}$ & $0 \mathrm{c}$ \\
\hline \multicolumn{5}{|l|}{ B } \\
\hline \multicolumn{5}{|l|}{ Alternância } \\
\hline 25-15 & $45 \mathrm{c}$ & $44 \mathrm{~b}$ & $90 \mathrm{a}$ & $14 \mathrm{~b}$ \\
\hline $30-15$ & $70 \mathrm{~b}$ & $52 \mathrm{~b}$ & $93 \mathrm{a}$ & $14 \mathrm{~b}$ \\
\hline $30-20$ & $82 \mathrm{a}$ & $81 \mathrm{a}$ & $94 \mathrm{a}$ & $53 \mathrm{a}$ \\
\hline $35-15$ & $0 \mathrm{~d}$ & $0 \mathrm{c}$ & $0 \mathrm{~b}$ & $0 \mathrm{c}$ \\
\hline $35-20$ & $0 \mathrm{~d}$ & $0 \mathrm{c}$ & $0 \mathrm{~b}$ & $0 \mathrm{c}$ \\
\hline $35-25$ & $0 \mathrm{~d}$ & $0 \mathrm{c}$ & $0 \mathrm{~b}$ & $0 \mathrm{c}$ \\
\hline
\end{tabular}


porcentagem de germinação na temperatura alternada de $30-20^{\circ} \mathrm{C}$, sendo a germinação significativamente reduzida nas alternâncias de $30-15$ e $25-15^{\circ} \mathrm{C}$ (Tab. 2B).

As espécies de Xyris estudadas não germinaram nas temperaturas constantes de 35 e $40^{\circ} \mathrm{C}$ e nas alternâncias em que foi incluída a temperatura de $35^{\circ} \mathrm{C}$ (Tab. 2A, 2B). As sementes de X. cipoensis também não germinaram a $15^{\circ} \mathrm{C}$ (Tab. $\left.2 \mathrm{~A}\right)$.

As quatro espécies de Xyris apresentaram maior tempo médio de germinação na temperatura constante de $15^{\circ} \mathrm{C}$ e nas alternâncias de $25-15$ e $30-15^{\circ} \mathrm{C}$, com exceção de $X$. platystachia que apresentou germinação mais rápida nas temperaturas alternadas de $25-15$ e $30-15^{\circ} \mathrm{C}$. O tempo médio de germinação foi menor nas temperaturas constantes de 20 a $30^{\circ} \mathrm{C} \mathrm{e}$ na alternância $30-20^{\circ} \mathrm{C}$ (Tab. 3).

\section{Discussão}

Devido à rápida redução no teor de água durante a fase final da maturação, a maioria das sementes apresenta de 5 a $20 \%$ de água da sua massa total (Bradbeer 1988). O teor de água das sementes investigadas neste estudo está dentro desta faixa, variando de 11 a $14 \%$, o que as caracteriza como ortodoxas. De acordo com Bewley e Black (1994), sementes ortodoxas mantêm baixo índice de umidade a fim de permanecerem viáveis por um longo período, germinando apenas quando as condições ambientais forem favoráveis à sobrevivência da plântula. $\mathrm{O}$ baixo teor de água destas sementes, além de limitar a germinação, é fundamental para evitar a deterioração das mesmas pelo ataque de microrganismos (Barbedo \& Marcos Filho 1998).

O tamanho das sementes possui relação direta com os processos de germinação, crescimento e estabelecimento das plântulas (Harper et al. 1970; Leishman et al. 1992). Segundo Harper et al. (1970), existe uma relação entre o tamanho das sementes e a necessidade de luz para germinação. Espécies com sementes pequenas geralmente requerem luz para a germinação (Venable \& Brown 1988; Pons 1992), o que resulta no impedimento do processo germinativo em profundidade no solo. Nesses casos a plântula apresenta dificuldade de emergir quando enterrada em conseqüência das reservas limitadas (Pearson et al. 2003), que seriam esgotadas antes que a plântula alcance a superfície do solo e inicie o processo fotossintético (Harper 1977; Bewley \& Black 1994). As espécies de Xyris apresentam sementes pequenas e fotoblásticas positivas, sendo capazes de germinar apenas nas camadas superficiais do solo, onde são expostas à luz, que é necessária para a quebra da fotodormência. Outros estudos mostram que sementes pequenas de espécies de campos rupestres apresentam comportamento fotoblástico (Sá e Carvalho \& Ribeiro 1994; Garcia \& Diniz 2003), o que sugere um padrão germinativo para as espécies desse tipo de ambiente.

$\mathrm{Na}$ maioria das sementes a temperatura influencia a velocidade e a porcentagem de germinação, pois altera a velocidade de absorção de água e das reações metabólicas das reservas necessárias para a sobrevivência da plântula (Baskin \& Baskin 1988; Bewley \& Black 1994). Segundo Ferreira et al. (2001),

Tabela 3. Tempo médio de germinação (em dias) de sementes de Xyris L., submetidas a temperaturas constantes (luz branca contínua) (A) e alternantes (fotoperíodo de $12 \mathrm{~h} / \mathrm{luz}$ ) (B). Letras iguais nas colunas não diferem entre si ( $\mathrm{P} \leq 0,05)$. (Média \pm desvio padrão).

A

\begin{tabular}{ccccc} 
& \multicolumn{4}{c}{ Espécie } \\
\cline { 2 - 5 } Temperatura $\left({ }^{\circ} \mathrm{C}\right)$ & X.cipoensis & X. longiscapa & X.platystachia & X. trachyphylla \\
\hline Constante & & & & \\
15 & - & $17,3 \pm 0,4 \mathrm{a}$ & $16,2 \pm 0,2 \mathrm{a}$ & $16,7 \pm 1,1 \mathrm{a}$ \\
20 & $9,1 \pm 0,8 \mathrm{a}$ & $8,6 \pm 0,5 \mathrm{bc}$ & $5,6 \pm 1 \mathrm{~b}$ & $9,5 \pm 0,1 \mathrm{~b}$ \\
25 & $9,2 \pm 0,8 \mathrm{a}$ & $9,6 \pm 0,4 \mathrm{~b}$ & $4,7 \pm 0,1 \mathrm{~b}$ & $6,7 \pm 0,3 \mathrm{c}$ \\
30 & $9,0 \pm 0,4 \mathrm{a}$ & $7,7 \pm 0,9 \mathrm{c}$ & $4,7 \pm 0,1 \mathrm{~b}$ & $5,7 \pm 0,2 \mathrm{c}$ \\
\hline
\end{tabular}

B

Alternância

25-15

$30-15$

$14,9 \pm 1,1 \mathrm{a}$

$13,6 \pm 0,5 \mathrm{a}$

$30-20$

$18,4 \pm 0,7 \mathrm{a}$
$14,3 \pm 1,5 \mathrm{~b}$
$8,7 \pm 0,8 \mathrm{c}$

$7,1 \pm 0,8 \mathrm{a}$
$6,6 \pm 0,1 \mathrm{ab}$
$5,6 \pm 0,1 \mathrm{~b}$

$17,6 \pm 3,2 \mathrm{a}$

$13,9 \pm 9,3$ a

$9,9 \pm 0,7 \mathrm{~b}$

$8,7 \pm 1,2 b$ 
o tempo médio de germinação é um índice que avalia a rapidez de ocupação de uma espécie em seu ambiente. As sementes das espécies investigadas apresentaram germinação mais lenta e decréscimo no percentual de germinação na temperatura de $15^{\circ} \mathrm{C}$ e alternadas com $15^{\circ} \mathrm{C}$, com exceção de $X$. platystachia $(25-15$ e 30 $15^{\circ} \mathrm{C}$ ). As sementes expostas às temperaturas de $35 \mathrm{e}$ $40^{\circ} \mathrm{C}$ apresentaram coloração escura e não germinaram quando transferidas para $20^{\circ} \mathrm{C}$ (dados não apresentados), o que indica perda da viabilidade nessas temperaturas. É conhecido que temperaturas elevadas alteram a permeabilidade das membranas e promovem desnaturação de proteínas necessárias à germinação, enquanto que baixas temperaturas retardam as atividades metabólicas, propiciando redução no percentual de germinação e atraso no processo germinativo (Simon et al. 1976; Bewley \& Black 1994).

A influência da temperatura no processo germinativo pode fornecer informações sobre o estabelecimento e regeneração de plantas em condições naturais (Cony \& Trione 1996). As sementes apresentam capacidade germinativa em limites bem definidos de temperatura, o que determina os padrões de distribuição das espécies (Thompson 1973; Labouriau 1983; Probert 1992). A germinação de $X$. longiscapa, X. platystachia e X. trachyphylla ocorreu em uma faixa de temperatura mais ampla (15 a $30^{\circ} \mathrm{C}$ ), condições também observadas para espécies de Eriocaulaceae (P.G. Oliveira, comunic. pessoal) e Velloziaceae (Garcia \& Diniz 2003), famílias com grande representatividade nos campos rupestres, assim como Xyridaceae. A espécie $X$. cipoensis apresentou faixa mais estreita de temperatura para a germinação $\left(20\right.$ a $\left.30^{\circ} \mathrm{C}\right)$, comportamento semelhante ao observado para Syngonathus venustus (P.G. Oliveira, comunic. pessoal), espécie de campos rupestres que também ocorre em ambientes alagados. $\mathrm{O}$ fato destas duas espécies ocorrerem exclusivamente em solos brejosos, sugere que no ambiente natural suas sementes estejam expostas a uma menor amplitude de temperatura, uma vez que a alta umidade interfere diretamente, diminuindo as flutuações de temperatura do solo (Egley 1986).

A germinação das sementes de muitas espécies é promovida em ambientes com temperaturas alternadas (Probert 1992). O requerimento de flutuações de temperatura é uma adaptação de espécies com sementes pequenas encontradas próximas à superfície do solo em ambiente aberto (Probert 1992), onde estão mais aptas a terem sucesso na germinação e no estabelecimento da plântula (Thompson 1974). Porém, apesar de possuírem sementes pequenas com fotoblastismo positivo restrito, as espécies de Xyris não apresentam exigências de amplitude térmica diária para germinação, uma vez que as flutuações de temperatura não aumentaram a germinação das sementes de $X$. cipoensis, X. longiscapa e X. platystachia e inibiram a germinação de $X$. trachyphylla, em relação às temperaturas constantes.

O conhecimento dos fatores que regulam pequenas populações é crítico para a conservação de espécies raras (Quilichini \& Debussche 2000; De Lange \& Norton 2004) e é fundamental para o entendimento da dinâmica das espécies vegetais e da comunidade (Brown et al. 2003). As espécies X. longiscapa e $X$. trachyphylla são freqüentes nos campos rupestres ao longo da Cadeia do Espinhaço e não são exploradas comercialmente. Xyris cipoensis e X. platystachia apresentam distribuição restrita aos campos rupestres de Minas Gerais e estão ameaçadas de extinção, por apresentarem populações pequenas submetidas à coleta predatória. Xyris cipoensis é endêmica da Serra do Cipó, encontrada apenas em algumas áreas de campos brejosos, em altitudes de 1.060 a $1.330 \mathrm{~m}$ (Giulietti et al. 1996). Os resultados sugerem que o endemismo de $X$. cipoensis pode ser determinado pelas características germinativas de suas sementes, uma vez que necessita de condições ambientais muito específicas para o início do processo germinativo e estabelecimento da plântula. Apesar disso, as populações de $X$. cipoensis não se encontram em áreas de preservação, o que torna a espécie mais suscetível à extinção, em virtude da grande exploração comercial. Desta forma, baseado nas informações obtidas, é necessário o desenvolvimento de um plano de manejo para a conservação dessas espécies, com atenção especial a $X$. cipoensis.

\section{Agradecimentos}

Os autores agradecem a Geraldo Rogério Faustini Cuzzuol, José Pires de Lemos Filho e Fábio Vieira, pela leitura crítica dos manuscritos e sugestões à redação do texto; à Maria das Graças Lapa Wanderley, pela identificação das espécies; à CAPES, pela bolsa concedida à primeira Autora.

\section{Referências bibliográficas}

Barbedo, C.J. \& Marcos Filho, J. 1998. Tolerância à dessecação em sementes. Acta Botanica Brasilica 12(2): 145-164. 
Baskin, J.M. \& Baskin, C.C. 1988. Germination ecophysiology of herbaceous plant species in a temperature region. American Journal of Botany 75: 286-305.

Bradbeer, J.W. 1988. Seed dormancy and germination. London, Blackie Academic \& Professional.

Bewley, J.D. \& Black, M. 1994. Seeds: physiology of development and germination. $2^{\text {nd }}$ ed. New York, Plenum Press.

Brown, J.; Enreght, N.J. \& Miller, B.P. 2003. Seed production and germination in two rare three common co-occurring Acacia species from south-east Australia. Austral Ecology 28(3): 271-280.

Cony, M.A. \& Trione, S.A. 1996. Germination with respect to temperature of two Argentinean Prosopis species. Journal of Arid Environments 33: 225-236.

De Lange, P.J. \& Norton, D.A. 2004. The ecology and conservation of Kurzea sinclairii (Myrtaceae), a naturally rare plant of rhyolitic rock outcrops. Biological Conservation 117: 49-59.

Egley, G.H. 1986. Stimulation of weed seed germination in soil. Reviews of Weed Science 2: 67-89.

Ferreira, A.G.; Cassol, B.; Rosa, S.G.T.; Silveira, T.S.; Stival, A.L. \& Silva, A.A. 2001. Germinação de sementes de Asteraceae nativas no Rio Grande do Sul, Brasil. Acta Botanica Brasilica 15(2): 231-242.

Garcia, Q.S. \& Diniz, I.S.S. 2003. Comportamento germinativo de três espécies de Vellozia da Serra do Cipó (MG). Acta Botanica Brasilica 17(4): 487-494.

Ghersa, C.M.; Benech-Arnold, R.L. \& Martinez-Ghersa, M.A. 1992. The role of fluctuating temperatures in germination and establishment of Sorghum hapelense. Regulation of germination at increasing depths. Functional Ecology 6: 460-468.

Giulietti, A.M; Wanderley, M.G.L.; Longhi-Wagner, H.M.; Pirani, J.R. \& Parra, L.R. 1996. Estudos em "semprevivas": taxonomia com ênfase nas espécies de Minas Gerais, Brasil. Acta Botanica Brasilica 10: 329-384.

Giulietti, N.; Giulietti, A.M.; Pirani, J.R. \& Menezes, N.L. 1988. Estudos de sempre-vivas: importância econômica do extrativismo em Minas Gerais, Brasil. Acta Botanica Brasilica 1: 179-194.

Harper, J.L.; Lovell, P.H. \& Moore, K.G. 1970. The shapes and size of seeds. Annual Review of Ecology and Systematic 1: 327-356.

Harper, J.L. 1977. Populations Biology of Plants. New York, Academic Press.

Kraus, J.E.; Sajo, M.G.; Dias Leme, C.L. \& Wanderley, M.G.L. 1994. Aspectos morfológicos do desenvolvimento pósseminal em espécies de Xyris L. (Xyridaceae). Hoehnea 21(1/2): 29-38.

Labouriau, L.G. 1983. A germinação das sementes. Secretaria Geral da Organização dos Estados Americanos. Washington, D.C.

Leishman, M.L.; Wright, I.J.; Moles, A.J. \& Westoby, M. 1992. The evolutionary ecology of seed size. Pp. 31-57 In: M. Fenner. Seed: The ecology of regeneration in plant communities. UK, CAB International.
Marques, A.R.; Garcia, Q.S.; Resende, J.L.P. \& Fernandes, G.W. 2000. Variations in leaf characteristics of two species of Miconia in the Brazilian cerrado under different light intensities. Tropical Ecology 41(1): 47-60.

Mendonça, M.P. \& Lins, L.V. 2000. Lista vermelha das espécies ameaçadas de extinção da flora de Minas Gerais. Fundação Biodiversitas \& Fundação ZooBotânica de Belo Horizonte (FZB-BH).

Pearson, T.R.H.; Burslem, D.F.R.P.; Mullins, C.E. \& Dalling, J.W. 2003. Functional significance of photoblastic germination in neotropical pioneer trees: a seed's eye view. Functional Ecology 17: 394-402.

Pons, T.L. 1992. Seed responses to light. Pp. 259-284. In: M. Fenner. Seed: the ecology of regeneration in plant communities. UK, CAB International.

Probert, R.J. 1992. The role of temperature in germination ecophysiology. Pp. 285-325. In: M. Fenner. Seed: the ecology of regeneration in plant communities. $\mathrm{UK}, \mathrm{CAB}$ International.

Quilichini, A. \& Debussche, M. 2000. Sees dispersal and germination patterns in a rare Mediterranean island endemic (Anchusa crispa Viv., Boraginaceae). Acta Oecologica 21(6): 303-313.

Rudall, P.J. \& Sajo, M.G. 1999. Systematic position of Xyris: flower and seed anatomy. International Journal Plant Science 160(4): 795-808.

Sá e Carvalho, C.G. \& Ribeiro, M.C. 1994. Efeitos do armazenamento e de reguladores de crescimento na germinação de Paepalanthus speciosus, Eriocaulaceae. Revista Brasileira de Botânica 17: 61-65.

Sajo, M.G.; Wanderley, M.G.L. \& Carvalho, L.M. 1995. Caracterização anatômica foliar para 14 espécies de Xyris L. (Xyridaceae) da Serra do Cipó, MG, Brasil. Acta Botanica Brasilica 9(1): 101-114.

Sampaio, I.B.M. 2002. Estatística Aplicada à Experimentação Animal. 2 ed. Belo Horizonte, Ed. Escola de Veterinária, UFMG.

Simon, E.W.; Minchin, A.; Mcmenamin, M.M. \& Smith, J.M. 1976. The low temperature limit for seed germination. New Phytologist 77: 301-311.

Smith, J. \& Downs, R. 1968. Xyridaceae. In: F.C. Hoehne (ed.). Flora Brasilica 9(2): 1-209.

Thompson, P.A. 1973. Geographical adaptation of seeds. Pp. 31-58. In: W. Heydecker. Seed ecology. London, Butterworths.

Thompson, P.A. 1974. Effects of fluctuating temperature on germination. Journal of Experimental Botany 25: 164-175.

Vázquez-Yanes, C. \& Orozco-Segovia, A. 1993. Patterns of seed longevity and germination in the tropical rainforest. Annual Review Ecology Systematic 24: 69-87.

Venable, D.L. \& Brown, J.S. 1988. The selective interactions of dispersal, dormancy and seed size as adaptations for reducing risk in variable environments. The American Naturalist 131(3): 360-384.

Zar, J.H. 1996. Biostatistical Analysis. $3^{\text {a }}$ ed. New Jersey, Prentice-Hall. 